\title{
TEACHER SUPPORT IN COVID-19 PANDEMIC TO DEVELOP BLENDED LEARNING DISRUPTIVE MODELS IN HIGHER EDUCATION
}

\author{
Francesco Floris, Alessio Genovese, Marina Marchisio, Fabio Roman and Matteo Sacchet \\ Department of Molecular Biotechnologies and Health Sciences, University of Turin, Italy
}

\begin{abstract}
Covid-19 pandemic has created significant changes in higher education institutions. After university lockdown, a transition from face-to-face learning to distance learning was unavoidable and several teachers and students had to approach new technologies. The DELTA (Digital Education for Learning and Teaching Advances) Research Group provided support to six degree programs at the University of Turin: each professor received specific trainings and the group constantly helped and checked the implementation of the online courses. In this paper the support provided during the emergency period has been analysed in order to evaluate the improvements in teaching methodologies, and to assess professors' transition to future blended learning disruptive models. The results show an important change in methodology for some courses, aiming at improving the online learning processes. The research data analysis and qualitative study about the usage of the Digital Learning Environment describe the courses' disruptive models. They are useful to understand which elements of the emergency response turned out to be positive and which ones to be unfavourable, in order to be able to redesign post-Covid higher education.
\end{abstract}

\section{KEYWORDS}

Blended Learning, Digital Learning Environment, Distance Teaching, Higher education, Online Teaching

\section{INTRODUCTION}

Over the last few years, education in universities and in schools has been characterized by a gradual introduction of new technologies and teaching methodologies based on blended learning, combining therefore a face-to-face component with digital and online learning (European Commission/EACEA/Eurydice, 2019).

The Covid-19 pandemic in Italy forced the implementation of emergency measures because of the lockdown of brick-and-mortar schools and universities since March, $5^{\text {th }} 2020$. To provide continuity of teaching activities, it became necessary to promote the transition from face-to-face or blended learning to a fully digital emergency approach. The implementation of new directives necessarily sped up the ongoing digitalisation process in schools and universities, binding all the users to an immediate and exclusive use of online learning tools. On one hand, the adoption of new ways of learning and teaching promoted the distribution of new technologies, while on the other hand, it often brought backlashes due to the sudden emergency situation, where both teachers and students had to deal with the acquisition of new digital skills. University professors needed to rapidly adapt whole courses to the new - and not yet mastered - educational approaches, while students had to adapt to new learning processes, little experienced before.

It should be noted that the new technologies introduced over time in university programs, such as Learning Management Systems (LMS), Interactive Whiteboard (IWB), digital laboratories, are not enough to guarantee the quality and effectiveness of teaching. They should be backed up with appropriate learning methodologies. Moreover, in Italy online learning plays a marginal role in university landscape, because only in a few cases online learning activities are officially allowed and approved, and this usually comes with some limitations. The degree programs are divided into four types defined by the Ministry of University and Research (MUR, 2019): conventional study programs, delivered entirely face-to-face with a maximum $10 \%$ of online learning; study programs with mixed modalities, for a maximum of $60 \%$ of online learning; study programs mainly at distance, with more than $60 \%$ of online learning; and study programs fully delivered online. The degree 
programs regarding some specific disciplines, such as Medicine and Architecture, must be by law conventional study programs, and almost all the degree programs of the public university fall into the first case.

At the University of Turin, during the Covid-19 crisis, the DELTA (Digital Education for Learning and Teaching Advances) Research Group, who does research in the field of Digital Education, provided support in this transition to professors of the Department of Molecular Biotechnologies and Health Sciences (DMBHS) and of the Interdepartmental University School of Strategic Sciences (SUISS), with a total of 6 degree programs, falling within the conventional study program typology. Before the pandemic, every degree program was already provided with a Digital Learning Environment (DLE), i.e. an online space shared between teacher and students for the availability and use of training (Badoni et al., 2011). Even if DLE was available to all the professors, it was rarely used effectively to improve learning processes or proposing online activities. The DLE provided to degree programs during the pandemic is based on a Virtual Learning Environment, a Moodle-Learning platform, combined with a web conference system, an Advanced Computing Environment (ACE) and an Automatic Assessment System (AAS). Concerning the courses belonging to the DMBHS, professors had required the addition of external tools integrated into the platform, which boasts several virtual laboratories. The DLE was already in use within SUISS (Spinello et al., 2019) and it was introduced within the DMBHS at the beginning of the academic year 2019-2020, to replace a former system: in November, two trainings on the usage of the AAS were provided, but the transition from the former platform to the new DLE was still ongoing. The support started with the setup of a number of specific trainings on the use of the DLE accompanied with a constant monitoring of the quality of the educational offer. The support was not only aimed to guarantee the teaching continuity using the DLE, but also to train the teachers to allow permanent and effective use of the new learning approaches.

In this paper we answer the following research questions:

- RQ1: How the support provided to the university professors in transition from a face-to-face approach to distance teaching and learning during Covid-19 emergency has been exploited to promote and spread the evolution from a traditional educational model to a blended learning disruptive model?

- RQ2: Which learning models were widely adopted by professors and how does the use of technology influence learning approaches?

In order to answer these questions, the research analysis data about the usage of the DLE and qualitatively study describes the courses disruptive approaches. The specific use of the technology in some courses offered by the 6 degree programs will be described through the SAMR model, and the most relevant methodologies will be identified, with reference to the classification proposed by the Christensen Institute. Section 2 describes the theoretical framework about blended learning models. Section 3 illustrates the research methodology and the support model adopted. Qualitative and quantitative results are presented in Section 4.

\section{THEORETICAL FRAMEWORK}

Even before the universities lockdown the use of technologies had been encouraged within the 6 degree programs to promote and spread blended learning approaches. Blended learning has been variously defined over time, due to the high number of different interpretations of this topic (Ossiannilsson, 2017). This paper relies on the characterisation proposed by the Christensen Institute, and in particular we use the different models described here to better analyse the activities proposed by professors. In this paper, we will adopt the definition of Blended learning as "a formal education program in which a student learns, at least in part through online delivery of content and instruction, with some element of student control over time, place, path, and/or pace, and at least in part in a supervised brick-and-mortar location away from home. The modalities along each student's learning path within a course or subject are connected to provide an integrated learning experience" (Christensen et al., 2013 p. 8).

\subsection{Theory of Hybrid Models in Blended Learning}

With reference to definition of blended learning mentioned above, it is possible to determine different models depending on different teaching approaches. These models are (Christensen et al., 2013):

- Rotation Model (which includes Station Rotation Model, Lab Rotation Model, Flipped Classroom, Individual Rotation) 
- Flex Model

- À la Carte Model

- Enriched Virtual Model

The first model can be considered as a blended learning approach as long as the educational activities switch between different learning methods, at least one being carried out online. This rotation can take place within the classroom itself (Station Rotation Model), between a classroom and a laboratory (Lab Rotation Model), between face-to-face guided activities and online contents available from the students outside the school (Flipped Classroom). The rotation can be personalised for each student, so that they do not need to approach to every option included in the rotation of the learning activities (Individual Rotation).

The Flex Model online learning is considered as the backbone, optionally referring to offline activities, and face-to-face component with the trainer is limited to the support activities. In the Â la Carte Model instead there is the possibility for the student to take some online courses in addition to the regular ones which take place in the brick-and-mortar school. In the last approach (Enriched Model) each course requires mainly fruition of online contents, but a periodical class attendance to lectures is required, too.

Which of these models can describe the learning activity before and during the Covid-19 emergency? Researchers are looking for disruptive models to propose for the future. The distinction between different blended learning models can be inserted in the more general theory of hybrid and disruptive models, coming from the economic field. A hybrid model upgrades old models through inclusion of new technologies, but it still represents a sustaining innovation, with the purpose of serving customers according to the original definition of performance. Disruptive innovations, unlike the sustaining ones, substantially transform the traditional idea of performance. Disruptive models have therefore several advantages compared to hybrid ones, such as the opportunity to approach new users excluded from the old system, the redesign of traditional models and the simplification of the quality of services. By analysing blended learning in the perspective of hybrid and disruptive models, blended learning might be considered as a hybrid model preceding the transition to the actual disruptive innovation of fully online learning. It is therefore important to point out that blended learning is considered here as a potential disruptive innovation in the field of education: relying on the different blended learning models described above, different teaching methods can assume both hybrid and disruptive characteristics.

\subsection{SAMR Model in Blended Learning}

The analysis of educational methodologies also concerns the role of technologies in planning the learning activities. We adopted the SAMR model to classify educational materials provided during Covid-19 emergency according to the technologies involved. This model includes 4 different approaches to new technologies (Puentedura, 2013):

- Substitution: technology acts as a direct substitute to traditional methods and resources

- Augmentation: technology acts as a direct substitute with functional improvements in the design of teaching activities

- Modification: technology affects the redesign of the learning task, with a significant modification

- Redefinition: technology allows to create new tasks, inconceivable in the traditional face-to-face model

As in the former theory of hybrid and disruptive models, also the SAMR model is characterized by a progression, with every step representing an improvement in learning outcomes and students' engagement.

\section{METHODOLOGY}

We adopted two different research work methods: the first one concerning quantitative data, the second one concerning qualitative analysis. We initially extracted the most relevant data on the use of the DLE: in fact, it allows to collect different typologies of reports on the activities of the platform, such as views, usage and interaction with the different types of resources or of activities. Our group exploited these reports both while monitoring teachers in distance learning activities and, subsequently, in the elaboration of the collected data. 
Data analysis was guided by the identification of different indicators: accessibility, availability, interactivity, flexibility, personalisation, completeness. The research group classified each type of resource according to such indicators in order to define the general trend of teachers' choices. Quantitative analysis has been deepened by a qualitative approach considering the supervision activity. In fact, support activities and constant monitoring of professors and courses on the DLE provided this study with relevant examples of distance learning approaches. A qualitative analysis of courses with reference to the SAMR model was performed, with the same purpose of detecting the suitability of different distance learning methodologies to a fully online approach. The consistent dialogue with professors gave us further elements to consider.

It was possible to recognize the main critical issues while analysing courses during the emergency. In critical cases, the support activity often influenced the choice of different materials, opting, for instance, for more interactive ones.

\subsection{Support Activity Provided}

The support group is composed mainly of researchers and instructional designers who are used to online learning methodologies and to the management of DLE (Marchisio et al., 2019). All components are administrators of the two platforms of DMBHS and SUISS and have the possibility to check all the courses and to manage users' accreditation. The group trained and supported professors to develop necessary digital skills and capability in planning an online course and in implementing it.

We provided training courses inside the DLEs, where self-learning materials and recorded online lectures dedicated to the teachers were uploaded. During the first days after university and school lockdown, free access basic trainings were scheduled. From March to June more than 15 hours of synchronous training were provided, concerning the management of the DLE, the creation of multimedia and interactive materials, the usage of the AAS. However, support was provided to professors mainly individually, through synchronous assistance (via call phone or VoIP systems) and asynchronous assistance (via e-mail or dedicated forum on the platform): more than 50 cases of synchronous assistance and more than 300 cases of asynchronous assistance. The former scheduled plan for face-to-face lectures was adapted into a new format which includes the distinction, specified by the teacher, between synchronous lectures (held via web conference system) and asynchronous modules (such as fruition of materials or activities uploaded). The adaptation of the schedule, especially in the cases of asynchronous activities, required attention to the correspondence between the hours required by the plan and quality and quantity of materials uploaded on the platform. In order to do so, a constant monitoring of every course, with in-depth periodical checks, was a relevant part of the work. Lastly, further reflections took place within internal meetings as departmental councils, with the aim to receive immediate feedback on the problematic aspects of DLE management by professors.

Training moments, personal support, course monitoring and classification of methodologies were not considered as different and subsequent phases. In fact, these aspects influenced each other: in particular, course monitoring improved support activities. Classification of use of technology and support events were two inseparable aspects of the research group activity.

\section{RESULTS}

Last February, before the emergency period, platforms of DMBHS and of SUISS registered 321 professors and 2028 students logged in. In a couple of weeks since the introduction of distance learning, after the creation of the missing courses, users counted 369 professors and 2355 students. The number constantly increased during the following months, due to the creation of new courses (sometimes external to the reference departments, like in the case of interfaculty courses). In July 2020, the DLE registered 201 active courses, 403 professors, 2763 students logged in. It should be noted that the growth is not exceptional, because many students and professors logged in just a few times in the first semester of a.y. 2019-2020.

Professors can choose among different types of resources and activities to share on the online platform, such as interactive lessons, books, automatic tests for formative and summative assessment, textual files, slides, videos, URLs to external links, forums, concept maps, folders of pictures, etc. It is interesting to have a look at the main types of resources and activities uploaded (Table 1 and Figure 1). The number of resources and 
activities uploaded on the two platforms during a.y. 2019-2020 totals 5094 in 201 active courses: only the most spread and significant typologies are reported.

Table 1. Main resources and activities uploaded during a.y. 2019-2020 by the 6 degree programs

\begin{tabular}{lll}
\hline Resources/Activities & Courses involved & Total numbers \\
\hline Resources (PDF files, PowerPoint, etc) & 112 & 2376 \\
Moodle Pages (videos, web pages, etc) & 42 & 514 \\
Labels (videos, section titles, etc) & 37 & 355 \\
URLs to external online contents (Youtube, news, etc) & 35 & 344 \\
Forums & 201 & 315 \\
Web Conference Sections & 56 & 219 \\
Test with Automatic Assessment & 51 & 169 \\
Assignments & 38 & 169 \\
Chats & 9 & 11 \\
\hline
\end{tabular}

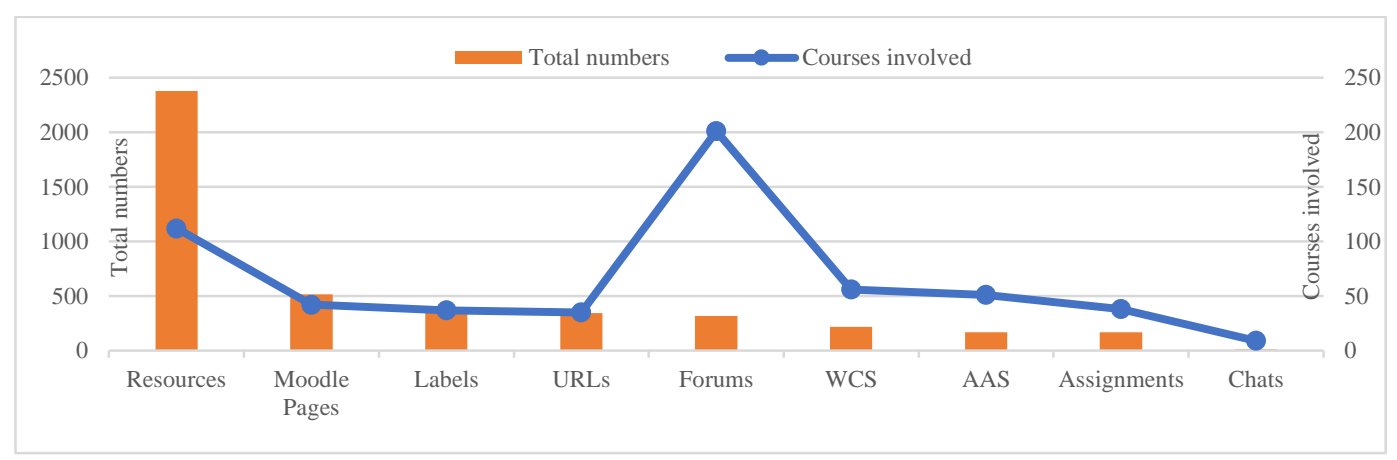

Figure 2. Main resources and activities uploaded during a.y. 2019-2020 by the 6 degree programs

In a few cases questionnaires, feedback tools, glossaries or other activities for collaborative learning were created. Interactive materials sometimes are created too, but in general this type of resources is less used than other ones, despite their relevance in online teaching, especially in STEM disciplines (Barana et al., 2019b). As it can be noticed, almost half is represented by static resources, mostly documents and slides. Video lessons can be uploaded on the platform through the tools Moodle page or labels. A relevant use of external resources can as well be observed in the number of URLs: many links refer to videos uploaded on platforms dedicated to multimedia materials sharing, significant of a quite spread use of the Open Educational Resources (OERs). This is very positive, because professors were trained on the importance of the use of OERs and the use of Creative Common Licences in order to share their materials.

An important specification is needed with regards to synchronous activities: even if they seem to be present in a low number of courses (a total of 219 activities in only 56 courses), these numbers do not take into account different systems external to the DLE, used by some of the teachers to virtually meet their students, and the fact that most professors used the same activity for all the live moments. Another interesting data concerns the use of forums: each course includes a default chat system for asynchronous communications, but the actual number of forum activities shows the importance of this system beyond the automatic announcement.

Through a comparison of the resources and activities provided in SUISS platform in a.y. 2019-2020 and the ones of a.y. 2018-2019, it can be noticed that while the total number of materials has more than doubled, the number of static resources has increased by $88 \%$. This is the smaller increase among the typologies presented in Table 1: for example, the use of OER through external URLs has increased by $460 \%$, video resources by $260 \%$. These data refer only to SUISS due to lack of data of previous years in DMBHS, and they show a change of needs and, during the emergency, support activities tried to understand and to answer new necessities. 


\subsection{Different Approaches to the use of Technology for Distance Learning}

In order to better analyse the methodologies adopted in online courses, it is first necessary to discuss how technology was used. Some typical examples of use of DLE during the emergency period seem useful to better describe the resources provided to the students through distance learning. With reference to the SAMR model, it should be noted that a significant case of "redefinition" model has not yet been identified: this achievement needs in fact a long and phased process of transition, difficult under extreme circumstances like Covid-19 emergency period.

\subsubsection{Online Learning as "Substitution"}

In certain cases, teachers initially did not upload any resource, while they only scheduled synchronous web conference activities, simply substituting face-to-face lectures with online live meetings where students are able to interact. These online sections do not cause any actual change in the educational offer, since they do not involve any improvement in the teaching methodology: the traditional setting simply shifted from the physical class to the online platform together with the synchronous explanation of the teacher. Technology acts therefore as a direct tool to substitute face-to-face lectures, without causing any change or improvement in the learning activity. The overlap between online teaching and synchronous activities also implies some recurrent disadvantages, such as the unavailability of a strong connection or the impossibility to use an appropriate device during classes, due to the schedule, for a part of the students. Training and support activities were therefore aimed at pointing out and discussing similar cases and highlighting other functions of DLE, monitoring the actual upload of the resources used in the synchronous meetings in order to guarantee the right to education to those students who were unable to attend classes.

\subsubsection{Online Learning as "Augmentation"}

In most part of the courses, students were provided, together with the resources, with uploaded video recorded lectures specifically projected for asynchronous fruition. These didactic materials allow the students to autonomously access resources with the further advantage of the teacher's oral explanation, which promotes a more effective comprehension and mastery. This use of DLE can be seen as a direct substitute to the face-to-face model but, in this case, resources and activities are projected to improve student's fruition through the possibility of self-management of materials and time and individualization of the learning process. To promote and spread the use of video lessons, decreasing the high number of synchronous activities, an important part of training activities was dedicated to video making and audio recording to be attached to slides presentation.

Other relevant examples of online learning as "augmentation" are the use of forum for questions and doubts about the disciplines to which students themselves were able to answer, and the use of an Automatic Assessment System for the formative evaluation, provided as online drills and followed by personalized feedback. This model of online learning brings about actual and relevant improvements to the learning processes, the most significant being the increase of students' engagement (Barana et al., 2020).

\subsubsection{Online Assessment as "Modification"}

The issue of distance exams and the necessity of new forms of assessment have been largely discussed during the lockdown of universities, and it would deserve an appropriate in-depth discussion. The University of Turin immediately provided professors with guidelines on the best evaluation systems, among which stands written testing and webcam checks. In our support and training professors were invited to redesign examination procedures in order to avoid cheating or other issues: some exams previously structured in open questions or multiple choice questions were redesigned as open book exams, focused on papers or works to be submitted. The advantage of open book exams, which allow the students to freely access educational materials and manage the execution time, consists in the possibility to avoid the difficulties presented by simultaneous tests, such as cheating or technical issues caused by synchronous use of online tools. Moreover, the use of specific tools which allow professors to add comments and assess students submissions was promoted. Since the assessment phase fully involves the didactic planning of a course, this is a relevant example of technology acting not only as direct substitute with functional improvements, but also as actual "modification" of traditional methodologies. Professors were invited to use also formative assessment and to use the examples of final assessments available on the platform in order to help students to better prepare for them (Barana et al., 2019a). 


\subsection{Adopted Methodologies towards a Flex Model}

With reference to the former analysis, which blended learning model better fits the learning methods proposed before and during the Covid-19 emergency? Before March 2020, digital support activities were already provided, but they were not very appealing: most of the courses were structured with the traditional face-to-face model and the use of technology often did not effectively influence learning methodologies. This marginality did not of course affect all courses: exceptions are represented by Mathematics and Physics modules of the three master's degree programs offered by DMBHS and SUISS. These courses were based on face-to-face teaching but a key point of the educational offers concerned also the use of the ACE and included a large use of interactive resources and activities in the DLE: several lectures were dedicated to practicing in a computer lab, and the study of a real problem through the ACE is required and evaluated. Courses presenting similar learning methodologies can actually be ascribed to the blended learning Lab Rotation Model, with some points in common with the Flipped Classroom model, such as the follow-up activities proposed by some teachers. Blended learning models mentioned above can be described as functional hybrid models, since they combine traditional elements (face-to-face learning activities) with innovative components (online learning through the use of a DLE), and involve the same users of the traditional system.

With reference to the methodologies proposed during distance teaching, it is necessary to specify that they clearly cannot be defined exactly as blended learning models, since the face-to-face component is absent. However, it is useful to adopt these models to classify the online learning activity, in order to choose future methodologies. The use of DLE, as we observed, shares common aspects with the traditional face-to-face approach, in particular by transposing physical lectures to online learning through synchronous lessons: courses based on the use of technologies as a direct substitute tool can be ascribed to traditional models.

In order to classify methodologies in distance teaching, it is important to understand how traditional methods (synchronous activities substituting face-to-face lectures) and innovative methods are related. As observed, one of the results of support activities was to avoid a simple transposition of the face-to-face schedule into only synchronous moments. In fact, most of the courses provided the students with autonomous study resources uploading online materials and video lessons. However, we observed an actual need from both students and professors for moments of discussion. Different tools can meet this need: forum activities were strongly recommended, and also a periodic (moderate) use of synchronous meetings, via chat or via web conference, was suggested. Therefore, almost all the courses included activities and resources available to students for autonomous learning, with the possibility of discussion moments with the teacher. Synchronous sections were a moment of discussion. With regards to the resources, with the help of training activities we were able to encourage professors to reconsider the phase of elaboration of educational material: a relevant example concerns the use of video lessons, mentioned above, not only delivered as a single video recorded but divided into shorter and well-structured unities, more accessible to students.

The blended learning model that better represents the methodology described above is the Flex model: students have maximum autonomy in planning and personalising learning processes, but they can benefit from necessary moments of discussion with the professor. In the characterization of the Christensen institute, the Flex Model is a disruptive model: it evolves the idea of students sitting in a brick-and-mortar classroom and not being able to manage their time, place, and path of learning. Even when considering it with its actual face-to-face component, including offline activities and discussions with the teacher, this model is still suitable for possible students who might encounter difficulties in attending face-to-face or hybrid courses.

A necessary component for an effective fruition of this methodology by students is a strong personal layout planning of the online course, together with a general well-structured organisation of the activities and resources, and with a desirable aware use of OERs. The support group contributed to the development of such skills in professors, with some significantly positive feedback.

With the help of instructional experts' suggestions, many professors managed to design their modules according to Flex Model methods. Someone took it even further: the teachers who learned how to use the AAS are able to use innovative assessment methodologies, such as the formative ones. As we observed, drills were provided, not only based on automatic assessment but also on multiple attempts and personalized feedback: in courses that provided such activities, students' participation was very high. An example is the Molecular Immunology course, where at least $60 \%$ of students took part in the latest optional tests. Some professors promote formative assessment for supporting autonomous studying and training in view of the exam. In such cases, personal assistance proved to be fundamental for positive outcomes. 


\section{CONCLUSION}

Through the support and training provided to the academic staff of 6 degree programs during the Covid-19 emergency, we observed different approaches to the use of technology (using the SAMR model), and we promoted a common disruptive model of blended learning in the courses provided. The forced and immediate use of new technologies caused some relevant changes in teaching methodologies: synchronous activities were initially largely spread and tended to maintain the structure of traditional face-to-face lectures as much as possible. After the support activities, both professors and students developed important skills about new methodologies, which can be ascribed to the Flex Model, which was eventually the most used learning model (RQ2). Thus, support activity was able to promote and spread the evolution of the educational model not only through technical assistance, but also, as we observed, through the proposal of innovative tech and confrontation with professors (RQ1).

It can be stated that almost all the teaching staff has acquired an open-minded approach and awareness in the use of disruptive teaching methods. It is appropriate to wonder how the emergency will influence the following months of stabilization and the future of learning methods, and what the better strategies are in order to provide necessary support and training for the gradual development of functional learning models for the best learning outcomes. In this perspective, it is conceivable and desirable to maintain disruptive mixed teaching models by providing constant support. For the development of disruptive learning methods in schools, the creation of "a team within the school that is autonomous from all aspects of the traditional classroom" is indicated by Christensen as the first step (Christensen et al., 2013p. 38). During the emergency, the DELTA Research Group actually played the role of an independent support group. The experience described in this paper shows how beneficial and effective an instructional team can be, not only for technical assistance but also for monitoring the activities and for suggestions concerning possible improvements of the online components of the courses, with potential benefit on students' outcomes and engagement. The cases presented in this paper are relevant examples of a support and research work aimed at achieving a deeper awareness.

Finally, it would be useful to deeper investigate feedback and issues concerning students' and professors' experience of management of distance learning: structured questionnaires dedicated to both professors and students are already available on DLE, and the analysis of results is still ongoing.

\section{REFERENCES}

Baldoni M. et al., 2011. HAP-Moodle: una soluzione open-source per l'High-Availability e la performance applicata a Moodle. E-learning con Moodle in Italia: una sfida tra passato, presente e futuro. Seneca Ed., Torino, pp. 213-226.

Barana A. et al., 2019a. Empowering engagement through automatic formative assessment, Proceedings of 2019 IEEE 43rd Annual Computer Software and Applications Conference, Milwaukee, Wisconsin, USA, pp. 216-225

Barana A. et al., 2019b. The role of an advanced computing environment in teaching and learning mathematics through problem posing and solving, 2019, eLearning and software for Education Conference, Bucharest, Romania, pp. 11-18

Barana A. et al., 2020. Empowering Engagement in a Technology-Enhanced Learning Environment, Project and Design Literacy as Cornerstones of Smart Education, Vol. 158, Springer, pp. 75-77

Christensen C. et al., 2013. Is K-12 blended leaning disruptive? An introduction to the theory of hybrids. Retrieved from: https://www.christenseninstitute.org/wp-content/uploads/2014/06/Is-K-12-blended-learning-disruptive.pdf

European Commission/EACEA/Eurydice, 2019. Digital Education at School in Europe. Eurydice Report. Luxembourg: Publications Office of the European Union

Marchisio et al., 2019, Instructional design to "train the trainers": the start@ unito project at university of Turin, 2019, Multi Conference on Computer Science and Information System, MCCSIS 2019 - Proceedings of the International Conference on e-Learning 2019, pp. 195-202

MUR, 2019. Decreto Ministeriale n. 989 del 25/10/2019 relativo alle Linee Generali di indirizzo della programmazione delle università 2019-2021

Ossiannilsson E., 2017. Blended Learning State of the Nation. Proceedings of the 10th International Conference on Computer Supported Education (CSEDU 2018), Funchal, Portugal, pp. 541-547

Puentedura R. R., 2013. SAMR: Moving from enhancement to transformation. Retrieved from http://www.hippasus.com/rrpweblog/archives/000095.html

Spinello E. et al., 2019. A full spectrum lifelong e-learning project for the army, 15th International Scientific Conference eLearning and Software Education, Bucharest, Romania, pp. 152-158 